\title{
Sympathetic nerve-derived ATP regulates renal medullary vasa recta diameter via pericyte cells: a role for regulating medullary blood flow?
}

\section{Crawford ${ }^{\dagger}$, S. S. P. Wildman ${ }^{*}{ }^{\dagger}$, M. C. Kelly, T. M. Kennedy-Lydon and C. M. Peppiatt-Wildman*}

Urinary System Physiology Unit, Medway School of Pharmacy, The Universities of Kent and Greenwich, Chatham Maritime, Kent, UK

\section{Edited by:}

Bellamkonda K. Kishore, University of Utah Health Sciences, USA

\section{Reviewed by:}

Roger Evans, Monash University, Australia

Volker Vallon, University of California San Diegio, USA

Tianxin Yang, University of Utah, USA

\section{${ }^{*}$ Correspondence:}

S. S. P. Wildman and C. M. Peppiatt-Wildman, Urinary System Physiology Unit, Medway School of Pharmacy, The Universities of Kent and Greenwich at Medway, Anson Building, Central Avenue, Chatham Maritime, Chatham, Kent, ME4 4TB, UK

e-mail: s.s.wildman@kent.ac.uk;

c.m.peppiatt@kent.ac.uk

${ }^{\dagger}$ Joint first authors.
Pericyte cells are now known to be a novel locus of blood flow control, being able to regulate capillary diameter via their unique morphology and expression of contractile proteins. We have previously shown that exogenous ATP causes constriction of vasa recta via renal pericytes, acting at a variety of membrane bound P2 receptors on descending vasa recta (DVR), and therefore may be able to regulate medullary blood flow (MBF). Regulation of MBF is essential for appropriate urine concentration and providing essential oxygen and nutrients to this region of high, and variable, metabolic demand. Various sources of endogenous ATP have been proposed, including from epithelial, endothelial, and red blood cells in response to stimuli such as mechanical stimulation, local acidosis, hypoxia, and exposure to various hormones. Extensive sympathetic innervation of the nephron has previously been shown, however the innervation reported has focused around the proximal and distal tubules, and ascending loop of Henle. We hypothesize that sympathetic nerves are an additional source of ATP acting at renal pericytes and therefore regulate MBF. Using a rat live kidney slice model in combination with video imaging and confocal microscopy techniques we firstly show sympathetic nerves in close proximity to vasa recta pericytes in both the outer and inner medulla. Secondly, we demonstrate pharmacological stimulation of sympathetic nerves in situ (by tyramine) evokes pericyte-mediated vasoconstriction of vasa recta capillaries; inhibited by the application of the P2 receptor antagonist suramin. Lastly, tyramine-evoked vasoconstriction of vasa recta by pericytes is significantly less than ATP-evoked vasoconstriction. Sympathetic innervation may provide an additional level of functional regulation in the renal medulla that is highly localized. It now needs to be determined under which physiological/pathophysiological circumstances that sympathetic innervation of renal pericytes is important.

Keywords: pericytes, ATP release, P2 receptors, vasa recta, vasoconstriction, kidney slice

\section{INTRODUCTION}

Dogma suggests that arterioles and precapillary sphincters are the key regulatory mechanisms of blood flow at the tissue level, however it is now becoming widely accepted that an additional locus of local blood flow regulation exists- that being pericyte cells found on the capillaries themselves (Peppiatt-Wildman, 2013). Pericyte cells are smooth muscle-like cells, comprised of a cell body and long processes, which run along and wrap-around the capillary, and possess the contractile proteins $\alpha$-smooth muscle actin and myosin. Pericytes have the ability to constrict and dilate capillaries, via their processes, following exposure to a number of vasoactive agents including adenosine $5^{\prime}$-triphosphate (ATP) (Peppiatt et al., 2006; Crawford et al., 2012; Peppiatt-Wildman, 2013).

Pericytes have been identified on capillaries in many organs of the body, including the kidney. Within the kidney, pericytes are primarily located on descending vasa recta (DVR) capillaries (Park et al., 1997). DVR extend from the renal cortex toward the outer and then inner medulla, with the highest density of pericytes being observed in the outer medulla (Park et al., 1997; Crawford et al., 2012). It has been suggested that renal pericytes play an integral role in regulating renal medullary blood flow (MBF), which in itself is essential to satisfy the conflicting demands of preserving the cortico-medullary gradients of $\mathrm{NaCl}$ and urea, while maintaining adequate oxygen and nutrient delivery, as well as metabolic clearance (Kennedy-Lydon et al., 2013). As such it has been further proposed that dysregulation and/or dysfunction of the pericyte cells themselves may account and/or contribute to a number of renal pathologies (Crawford et al., 2012; Peppiatt-Wildman, 2013).

Using our live tissue slice model (i.e., where vasa recta and pericytes are located in situ), we previously demonstrated that exogenous ATP, a well-documented vasoactive transmitter, causes constriction of vasa recta via renal pericytes (Crawford et al., 2011). We further demonstrated that exogenous noradrenaline (NA) causes constriction of DVR via renal pericytes (Crawford et al., 2012), and ATP acts via a variety of pericyte-membrane bound P2 receptors (nee purinoceptors), (Crawford et al., 2011). 
Consequently, this study added to an already substantial wealth of scientific publications that propose key physiological roles for extracellular ATP and P2 receptors (i.e., purinergic signaling) in the kidney; specifically in this case, a key role for purinergic signaling in regulating renal MBF (Crawford et al., 2011).

Given the increasing interest in renal purinergic signaling it is perhaps not surprising that various sources of endogenous extracellular ATP have been proposed, including renal epithelial, endothelial and red blood cells, in response to stimuli such as: mechanical stimulation (e.g., stretch, increased flow rate, and osmotic swelling), local acidosis, hypoxia, exposure to various hormones (e.g., vasopressin and aldosterone), and to a lesser extent from sympathetic nerve varicosities (Bodin et al., 1991; Sprague et al., 1996; Schwiebert, 2001; Jans et al., 2002; Burnstock and Knight, 2004; Praetorius et al., 2005; Wildman et al., 2009).

Extensive sympathetic innervation of the kidney has previously been shown, however the innervation reported has focused around the proximal tubules, distal tubules and ascending loop of Henle (Dibona, 2000). Sympathetic nerves follow the arterial vasculature from the renal artery to the outer medullary portions of the descending (and ascending) vasa recta, but have not been reported in the inner medulla or papilla regions (Barajas et al., 1992). Electron microscopy has demonstrated that sympathetic nerve varicosities come into close contact with effector cell membranes (both tubular and vascular cell) in the renal cortex, where released transmitters presumably act on receptors expressed by the effector cells. It is likely that a balance between sympathetic nerves and intra-renal effector cells is essential for optimal renal function (Dibona, 2005). Both ATP and NA are co-released from sympathetic nerve varicosities (Burnstock, 1990). Sympathetic nerve stimulation has been shown to induce the release of ATP and NA in human cortical kidney slices (Rump et al., 1996). In vivo studies in rats and rabbits have shown that cortical blood flow (CBF) and total renal blood flow (RBF) are regulated in part, by renal sympathetic nerves, whereby electrical stimulation causes constriction of arteries and arterioles at higher frequencies (Rudenstam et al., 1995; Leonard et al., 2000). We now know quite a lot about the effect of sympathetic nerve stimulation on MBF (Walkowska et al., 2005; Eppel et al., 2006a,b; Johns et al., 2011) but some important questions remain, for example are vasa recta pericytes innervated, can neurotransmitter release from nerves associated with said pericytes induce vasoconstriction, and does ATP contribute to this vasoconstriction?

Given that renal pericytes are likely key regulators of $\mathrm{MBF}$, and that purinergic (and noradrenergic) signaling potentially plays a role in the regulatory process, we hypothesize that sympathetic nerves may be an additional source of ATP acting at renal pericytes to regulate $\mathrm{MBF}$. In the current study we use our novel kidney tissue slice model in combination with immunohistochemistry to determine the proximity of vasa recta pericytes to sympathetic nerves in the renal medulla. In addition we investigate the effect of pharmacologically stimulating the release of transmitter substances (ATP and NA) from sympathetic nerves on pericyte activity. Identification of a new pathway (i.e., sympathetic innervation) for the regulation of renal pericytes may inform studies seeking to delineate the elusive mechanisms underlying renal pathology.

\section{METHODS}

\section{TISSUE SLICING}

Animal experiments were conducted in accordance with United Kingdom Home Office Scientific Procedures Act (1986). Kidney tissue slices were obtained as previously described (Crawford et al., 2012). Adult male Sprague Dawley rats ( 250 g, purchased from Charles River UK Ltd., Kent, UK) were killed by cervical dislocation; their kidneys were immediately removed and placed in ice-cold physiological saline solution (PSS) bubbled with 95\% $\mathrm{O}_{2} / 5 \% \mathrm{CO}_{2}$ and prepared for slicing. Prior to slicing, the kidneys were de-capsulated and any renal artery remnants removed. A single kidney was secured on the slicing block of a vibratome tissue slicer (Leica model VT1200S, Leica Microsystems (UK) Ltd, Milton Keynes, UK), and submerged in a bath of ice cold PSS bubbled with $95 \% \mathrm{O}_{2} / 5 \% \mathrm{CO}_{2}$. PSS contained (mM) $100 \mathrm{NaCl}$, $5 \mathrm{KCl}, 0.24 \mathrm{NaH}_{2} \mathrm{PO}_{4}, 0.96 \mathrm{Na}_{2} \mathrm{HPO}_{4}, 10 \mathrm{Na}$ acetate, $1 \mathrm{CaCl}_{2}$, $1.2 \mathrm{MgSO}_{4}, 5$ glucose, $25 \mathrm{NaHCO}_{3}, 5$ pyruvate (Sigma-Aldrich Ltd, Dorset, UK). The $\mathrm{pH}$ was adjusted to 7.4 using $10 \mathrm{M} \mathrm{NaOH}$. The outer cortical dome region $(\sim 3 \mathrm{~mm}$ tissue) of the kidney was removed to expose the top of the renal medulla and serial $200 \mu \mathrm{m}$-thick coronal kidney slices (in which there was intact cortex and medulla) were cut. Slices were collected and maintained at room temperature in a holding chamber containing PSS, and bubbled with $95 \% \mathrm{O}_{2} / 5 \% \mathrm{CO}_{2}$ to conserve tissue viability. The slices to be used in "live" experiments were maintained for up to $3 \mathrm{~h}$ in the holding chamber, or slices were fixed with 4\% paraformaldehyde (PFA, Sigma-Aldrich Ltd, Dorset, UK) and used for immunohistochemistry.

\section{IMMUNOHISTOCHEMISTRY}

For experiments in which pericytes and sympathetic nerves were co-labeled, fixed kidney tissue slices were incubated with anti-NG2 (Calbiochem, Merck Millipore, Middlesex, UK) and anti-tyrosine hydroxylase (anti-TH; Vector Laboratories Ltd, Peterborough, UK) primary antibodies, respectively. The anti-NG2-labeled pericytes were probed with a fluorescent Alexa Fluor 555-conjugated secondary antibody (Invitrogen Life Technologies, Paisley, UK), as previously described (Crawford et al., 2012). The tyrosine-hydroxylase signal was amplified with a biotinylated secondary antibody and probed with a FITC-conjugated tertiary antibody (Fluorescent Avidin Kit, Vector Laboratories Ltd, Peterborough, UK). Kidney slices were mounted using Citiflour (Agar Scientific Ltd., Stanstead, UK). The medulla of fixed slices was imaged using a Zeiss LSM 510 laser scanning confocal microscope (Carl Zeiss Ltd., Welwyn Garden City, Hertfordshire, UK). FITC-conjugated secondary antibodies were excited at $488 \mathrm{~nm}$ and Alexa Fluor 555 -conjugated secondary antibody excited at $543 \mathrm{~nm}$. Emitted light was collected with the following filters: long-pass $560 \mathrm{~nm}$ (Alexa Fluor 555) and band-pass 505-550 nm (FITC). Pericytes and sympathetic nerves were imaged in both the inner and outer medulla. Z-stack images ( $40 \times$ magnification) were also obtained in order to measure the distance between nerves and pericytes. At least three z-stacks were obtained in both inner and outer medulla per slice. The distances between sympathetic nerves and the nearest pericyte, in both inner and outer medulla, were measured using Zeiss LSM image 
browser software (Carl Zeiss Ltd., Welwyn Garden City, Hertfordshire, UK).

\section{FUNCTIONAL EXPERIMENTS AND ANALYSIS}

Functional DIC imaging experiments in live tissue, investigating in situ pericyte-mediated changes in vasa recta diameter, were performed as previously described (Crawford et al., 2011, 2012). Live kidney slices were secured in an open-bath chamber using a purpose-built platinum slice anchor and transferred to the stage of an upright Olympus microscope (model BX51WI, Olympus Microscopy, Southend-on-Sea, UK). Kidney slices were continuously superfused ( $2.5 \mathrm{ml} / \mathrm{min} ; 1.25 \mathrm{ml}$ bath volume) with PSS, bubbled with $95 \% \mathrm{O}_{2} / 5 \% \mathrm{CO}_{2}$ and maintained at room temperature. Pericytes on the vasa recta capillaries (vasa recta were defined as $<10 \mu \mathrm{m}$ in diameter) were identified by their previously established "bump-on-a-log" morphology (Crawford et al., 2012), and DIC images were captured through an Olympus $60 \times$ water immersion objective (0.9 NA). Real-time video images of changes in vasa recta diameter were collected every second by an attached Rolera XR Charge Coupled Devise (CCD) camera and recorded using Image Pro Software (Media Cybernetics Inc., Marlow, UK). Kidney slices were superfused with PSS alone for $70 \mathrm{~s}$ to establish a baseline vessel diameter at the pericyte and non-pericyte site. Slices were then exposed to tyramine $[1 \mu \mathrm{M}$; for $\sim 150 \mathrm{~s}$, sufficient time to evoke neurotransmitter release (Glover et al., 1983)], ATP $(100 \mu \mathrm{M})$, suramin $(100 \mu \mathrm{M})$, or combinations of the agents, before being subjected to a PSS wash. Compounds were purchased from Sigma-Aldrich Ltd, Dorset, UK, and working solutions were prepared in oxygenated PSS.

Time-series analysis of live kidney slice experiments was carried out using the public domain software Image $(\mathrm{NIH}$, http://rsb.info.nih.gov.ij), as previously described (Crawford et al., 2012). For each experiment, both a pericyte site and a nonpericyte site were identified on a single vasa recta and the diameter of the vasa recta at both locations was measured every five frames for the duration of the experiment $(1$ frame $=1 \mathrm{~s})$. Each capillary acted as its own control; an average of the first five measurements was taken to represent the resting baseline diameter value, and expressed as $100 \%$, for both pericyte and non-pericyte sites. All subsequent diameter measurements were calculated and expressed as a percentage of the corresponding baseline value for both pericyte and non-pericyte sites as previously described (Crawford et al., 2012).

For all experiments, statistical significance was calculated using Student's $t$-test; $P<0.05$ (two tailed) was considered significant. Values are expressed as mean \pm SEM; sample size $(n)$ represents the number of pericytes ( 1 pericyte and non-pericyte site per kidney slice). All experiments were performed in at least three different animals. One vasa recta per kidney slice was used to ensure all vessels were "naïve" prior to exposure to any drug.

\section{RESULTS}

\section{SYMPATHETIC INNERVATION OF THE RENAL MEDULLA}

Pericytes and sympathetic nerves were labeled in the medulla of fixed kidney tissue slices using anti-NG2 and tyrosine hydroxylase $(\mathrm{TH})$ antibodies, respectively, as described in the methods section (Figures 1Ai, Aii). Pericytes were clearly defined by their
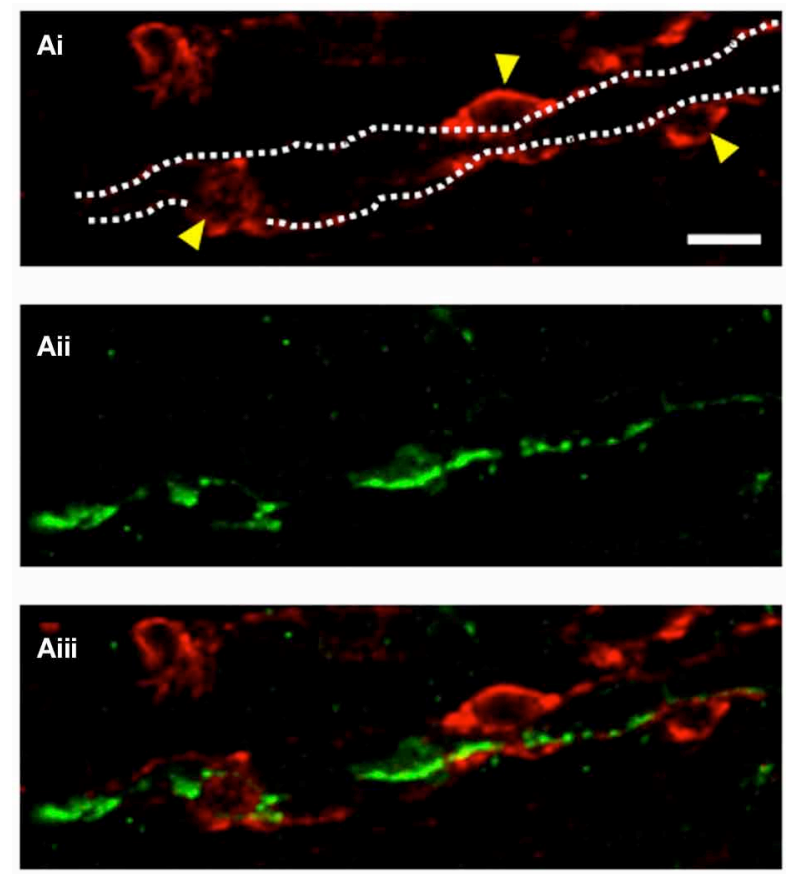

$\mathbf{B}$

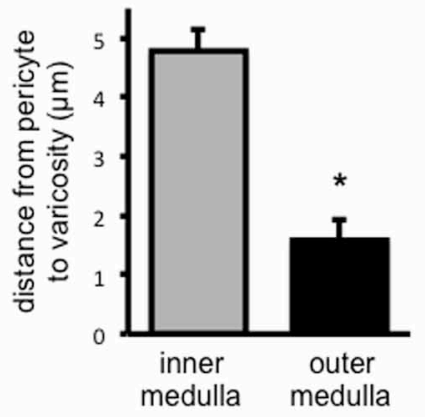

FIGURE 1 | Sympathetic nerves in close apposition to pericyte cells in the renal medulla. In (Ai), confocal image of fixed kidney slices (outer medulla region) incubated with anti-NG2, and probed with Alexa 555-conjugated secondary antibody (red) to label vasa recta pericytes and their processes. Pericyte cell bodies are highlighted (arrowheads), and the capillary wall is outlined (dotted lines). In (Aii), from the same image as in (Ai), sympathetic nerves were labeled with anti-tyrosine hydroxylase primary antibody, probed with Alexa 488-conjugated secondary antibody (green). In (Aiii), an overlay of images (Ai, Aii) shows sympathetic nerves running along vasa recta capillaries, in close proximity to pericytes. Scale bar $=10 \mu \mathrm{m}$. The distance between pericytes and the nearest sympathetic nerve varicosity was measured from confocal images using image browser software. In (B), the distance between pericyte and varicosity was significantly less in the outer medulla region $(n=21)$ when compared to the inner medulla $(n=21 ; * P<0.05)$

bump-on-a-log morphology (Figure 1Ai). Sympathetic nerves were consistently found running through the entire medulla region [i.e., both the outer $(n=21)$ and inner medulla $(n=19)$ ]. In all slices analyzed, sympathetic nerves were found, irrespective of localization in the medulla, to be in close proximity to vasa recta perciytes (Figure 1Aiii). Confocal z-stack images were used to follow nerves through the tissue, and the distance from pericyte 
to the nearest sympathetic nerve varicosity (defined by their nodule-like appearance) was measured using image browser software (see Supplementary Movie 1 for an example z-stack image). The distance from pericyte to the nearest sympathetic nerve varicosity was significantly less in the outer medulla $(1.58 \pm 0.35 \mu \mathrm{m}$, $n=21)$ compared to the inner medulla $(4.78 \pm 1.08 \mu \mathrm{m}, n=19$, ${ }^{*} P<0.05$, Figure 1B). Appropriate, parallel control experiments were carried out omitting anti-TH, anti-NG2, and/or biotinylated secondary antibodies, which confirmed an absence of fluorescent labeling.

\section{EFFECT OF TYROSINE-EVOKED NEUROTRANSMITTER RELEASE ON VASA RECTA DIAMETER}

Tyramine was superfused onto live kidney tissue slices in order to stimulate the release of sympathetic neurotransmitters (i.e., ATP and NA; via two distinct mechanisms; displacement from the axoplasm and subsequent vesicular release) from varicosities (Trendelenberg et al., 1987; Kirkpatrick and Burnstock, 1994) and the ability of tyramine-evoked neurotransmitter release to alter the diameter of subsurface vasa recta at both pericyte and non-pericyte sites was measured. Superfusion $(\sim 150 \mathrm{~s})$ of tyramine $(1 \mu \mathrm{M})$ caused a slowly-reversible vasoconstriction of vasa recta at pericyte sites (Figure 2A). During exposure to tyramine, vasoconstriction was significantly greater at pericyte sites $(3.9 \pm$ $0.9 \%, n=7)$ than at non-pericyte sites $\left(1.4 \pm 0.3 \%, n=7,{ }^{*} P<\right.$ 0.05 , Figure 2B). Maximum tyramine-evoked vasoconstriction occurred $\sim 550$ s $(534 \pm 115 \mathrm{~s}, n=7)$ after the cessation of tyramine superfusion (i.e., during tyramine-washout, Figure 2A). Maximum vasoconstriction, i.e., during tyramine-washout, at the pericyte site was $11.9 \pm 2.5 \%$ as compared to $3.0 \pm 0.6 \%$ at the non-pericyte site $\left(n=7,{ }^{*} P<0.05\right.$, Figure $\left.2 \mathbf{B}\right)$, and was significantly greater than that measured when tyramine was present in the bath ${ }^{* *} P<0.05, n=7$, Figure $2 B$ ). Complete reversibility (i.e., when vessel diameter returned to baseline) was seen $\geq 600 \mathrm{~s}$ from the start of tyramine-washout $(n=7)$. Using Poiseuille's law, the maximum tyramine-evoked vasoconstriction of $12 \%$, equates to a $\sim 1.7$-fold increase in vasa recta resistance and thus $\mathrm{a} \sim 40 \%$ decrease in blood flow (Peppiatt et al., 2006; Crawford et al., 2012).

Tyramine evokes the co-release of ATP and NA from sympathetic nerve varicosities. To investigate the proportion of ATP-mediated vasoconstriction vs. NA-mediated vasoconstriction, suramin was superfused onto the tissue slices, for the entirety of each experiment, in order to antagonize ATPactivated P2 receptors, including those present on renal pericytes. Superfusion (for $\sim 150 \mathrm{~s}$ ) of tyramine $(1 \mu \mathrm{M}$ ) following suramin pre-treatment, and in the continued presence of suramin $(100 \mu \mathrm{M})$, resulted in the maximum tyramine-evoked vasoconstriction (measured during tyramine-washout) being reduced by approximately $50 \%$ at pericyte sites ( ${ }^{* *} P<0.05$; Figure $2 \mathrm{C}$ ). The maximum tryamine-evoked vasoconstriction at pericyte sites, in the presence of suramin, was reduced to $5.8 \pm 1.7 \%$, and was still significantly greater than the change in vessel diameter recorded at non-pericyte sites $\left(2.1 \pm 0.7 \%,{ }^{*} P<0.05, n=6\right.$; Figure 2C). Incidentally, in 6 out of 10 experiments suramin $(100 \mu \mathrm{M})$ alone caused a modest vasoconstriction of vasa recta
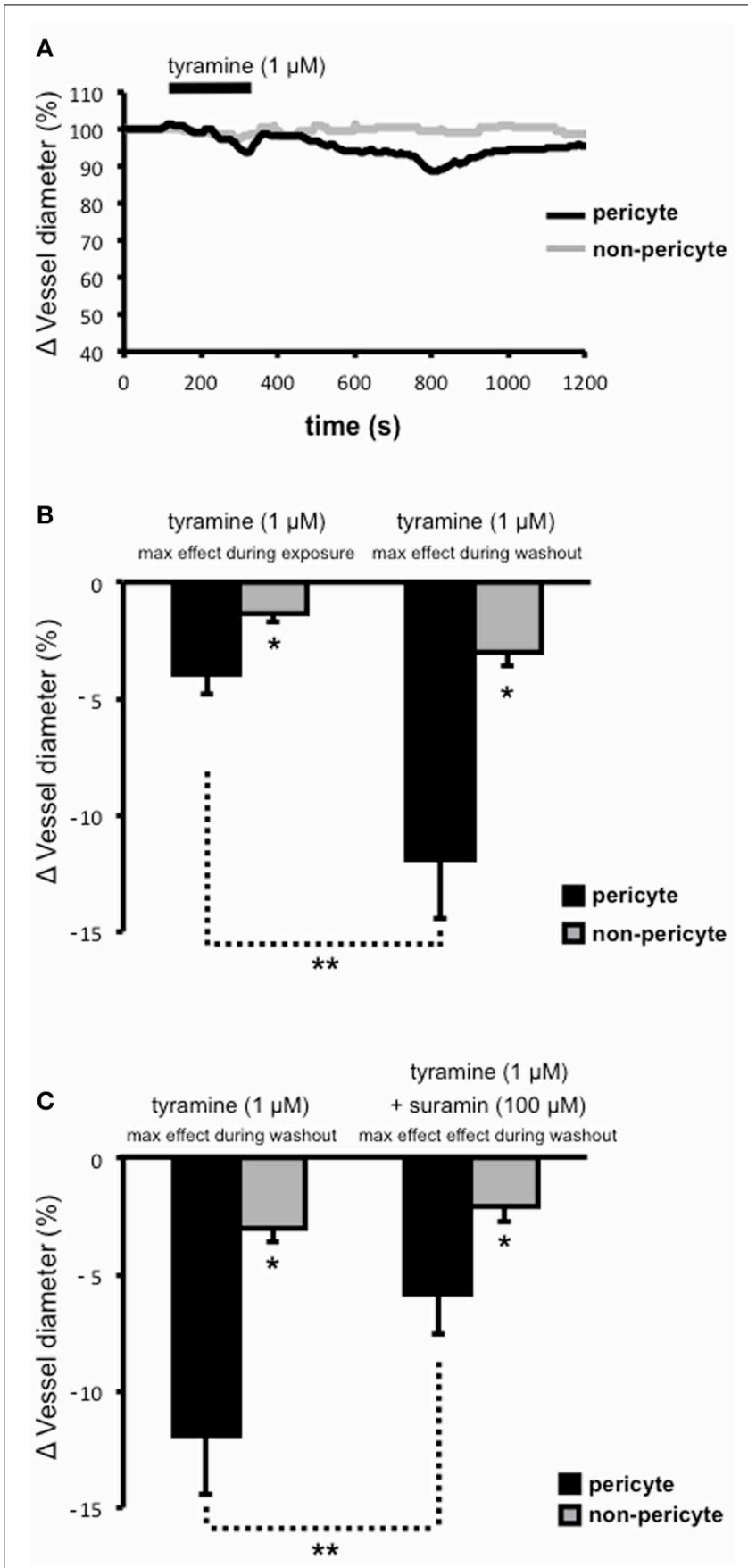

FIGURE 2 | Tyramine-evoked changes in vasa recta diameter. Live kidney slices were superfused with tyramine $(1 \mu \mathrm{M})$. A typical trace from a single experiment shows changes in vessel diameter over time at a pericyte (A, black line) and corresponding non-pericyte site (A, gray line). Mean data in bar graphs in (B), and (C), show a significantly greater change in vessel diameter at pericyte sites (black bars) compared to non pericyte sites (gray bars), both during tyramine $(1 \mu \mathrm{M})$ exposure and during the tyramine-washout period $(\mathbf{B}, * P<0.05, n=7)$. The constriction measured at pericyte sites was significantly greater during the tyramine washout step than that measured when tyramine was present in the bath $\left(\mathbf{B}^{* *} P<0.05\right.$, $n=7)$. The maximum tyramine-evoked constriction seen at pericyte sites was significantly inhibited by the presence of suramin $(C, 100 \mu \mathrm{M}$, ${ }^{* *} P<0.05, n=6$ ). 
at pericyte sites $(2-5 \%)$. Whilst suramin significantly altered the maximum tyramine-evoked constriction of vasa recta by pericytes, the presence of suramin did not alter the onset time, time to maximum effect, nor reversibility, of tyramine-evoked vasoconstriction (data not shown, $n=6$ ).

Extracellular ATP acts at pericytes to cause vasoconstriction of vasa recta (Crawford et al., 2011) and various sources of endogenous extracellular ATP have been proposed including from renal epithelial cells, endothelial cells, red blood cells, and sympathetic nerve varicosities. To investigate the significance/relative contribution of varicosity-derived ATP to ATPevoked, pericyte-mediated, vasoconstriction of vasa recta, the additive effects of tyramine and ATP $(100 \mu \mathrm{M})$, at a concentration previously shown to evoke the greatest constriction of vasa recta (Crawford et al., 2011), were investigated. Prolonged superfusion $(\sim 800 \mathrm{~s})$ of tyramine $(1 \mu \mathrm{M})$ caused a constriction of vasa recta at pericyte sites (Figure 3A) culminating in a maximum effect between 400-600s $(n=5)$. During exposure to tyramine, maximum vasoconstriction was significantly greater at pericyte sites $(7.8 \pm 3.6 \%, n=5)$ than at non-pericyte sites

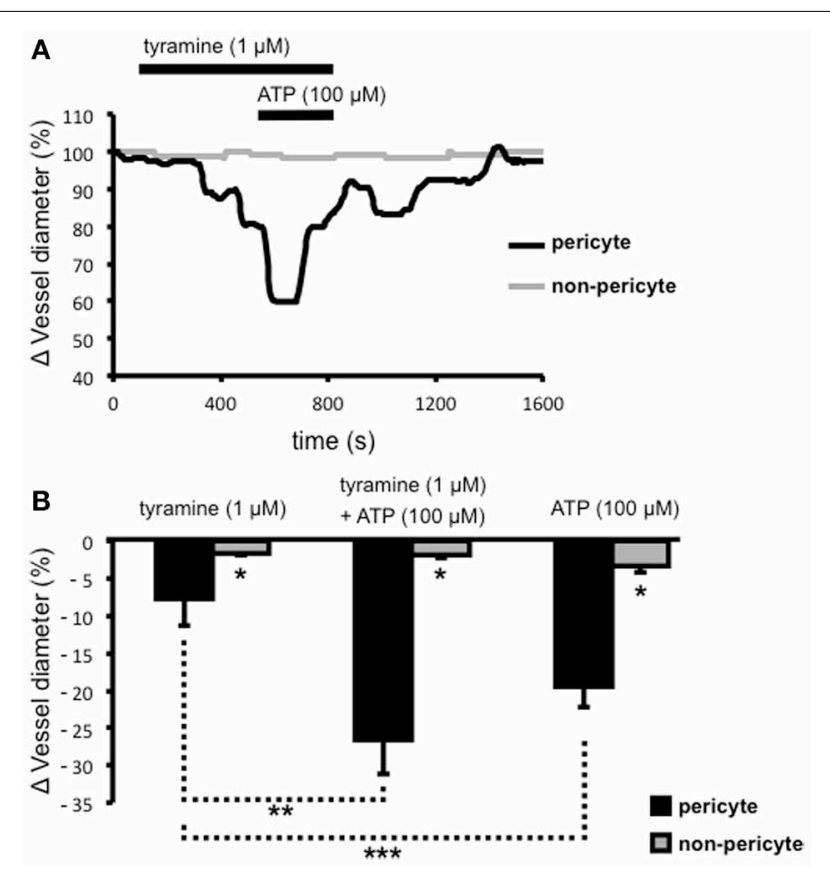

FIGURE 3 | The additive effects of ATP and tyramine on pericyte-mediated changes in vasa recta diameter. Live kidney slices were superfused with tyramine $(1 \mu \mathrm{M})$ and ATP $(100 \mu \mathrm{M})$; a typical trace from one of these experiments shows changes in vessel diameter over time at a pericyte (A, black line) and corresponding non-pericyte site (A, gray line). Mean data for these experiments show a significantly greater change in vessel diameter at pericyte sites (black bars) compared with non-pericyte sites (gray bars) under all experimental conditions ${ }^{*} P<0.05$, $n=5)$. The combined tyramine $(1 \mu \mathrm{M})$ and ATP $(100 \mu \mathrm{M})$-evoked constriction at pericyte sites was significantly greater than that observed during tyramine $(1 \mu \mathrm{M})$ incubation alone $\left(\mathbf{B}^{* *} P<0.05, n=5\right)$, and the pericyte-mediated constriction of vasa recta in the presence of exogenous ATP was significantly greater than that evoked by tyramine alone $(* * * P<0.05, n>5)$. $\left(1.6 \pm 0.3 \%, n=5,{ }^{*} P<0.05\right.$, Figure 3B). The co-application of ATP $(100 \mu \mathrm{M}$; for $\sim 200 \mathrm{~s})$ with tyramine $(1 \mu \mathrm{M})$ during the "maximum tyramine-evoked vasoconstriction period" caused a further increase $\left({ }^{* *} P<0.05\right.$, Figure 3B $)$ in the constriction of vasa recta at pericyte sites $(26.5 \pm 4.6 \%)$ that was significantly greater than at non-pericyte sites $(1.7 \pm 0.5 \%, n=5$, ${ }^{*} P<0.05$, Figure 3B). The exogenous ATP-evoked vasoconstriction rapidly desensitized in the presence of exogenous ATP and complete reversibility was seen $\geq 700 \mathrm{~s}$ from the start of tyramine/exogenous ATP-washout (Figure 3A). Superfusion (for $\sim 150$ s) of ATP $(100 \mu \mathrm{M})$ alone caused a reversible vasoconstriction of vasa recta at pericyte sites; maximum exogenous ATP-evoked vasoconstriction at the pericyte site was $19.4 \pm$ $2.8 \%$ as compared to $3.3 \pm 2.9 \%$ at the non-pericyte site $\left({ }^{*} P<\right.$ $0.05, n=11$, Figure 3B). The exogenous application of ATP $(100 \mu \mathrm{M})$ alone caused a significantly greater constriction than that observed with tyramine, at pericyte sites $\left(1 \mu \mathrm{M}\right.$, ${ }^{* * *} P<0.05$, Figure 3B).

\section{DISCUSSION}

The main findings of this investigation, using the live kidney slice model, revealed sympathetic nerves in both the outer and inner medulla regions of the kidney, and that pharmacological stimulation of sympathetic nerves in situ (by tyramine) evokes pericyte-mediated vasoconstriction of vasa recta capillaries. More specifically, we present evidence that (i) sympathetic nerve varicosities are in closer apposition to pericytes in the outer medulla, (ii) tyramine-evoked vasoconstriction of vasa recta by pericytes is significantly inhibited by the $\mathrm{P} 2$ receptor antagonist suramin, (iii) tyramine-evoked vasoconstriction of vasa recta by pericytes is significantly less than exogenous ATP-evoked vasoconstriction. Importantly, these novel findings reveal innervation of renal pericytes by sympathetic nerves, primarily in the outer medulla. This finding being ratified by previous studies, which describe innervation of medullary tubules (Dibona, 2000; Loesch et al., 2009). Moreover, functionally these findings demonstrate that sympathetic nerve-derived ATP, acting as a cotransmitter, is as effective as, or more effective than NA, in its ability to regulate vasa recta diameter, and thus MBF. As such, sympathetic innervation may provide an additional level of functional regulation in the renal medulla that is highly localized (see Figure 4).

\section{SYMPATHETIC NERVE VARICOSITIES IN CLOSE APPOSITION TO PERICYTES IN THE OUTER MEDULLA}

The current study identifies, for the first time, TH-labeled, sympathetic varicosities in both the outer and inner medulla regions of fixed rat kidney tissue (Figure 1). Our novel live kidney slice model in combination with confocal z-sectional imaging enabled us to observe the path of sympathetic nerves through the tissue, running in parallel to vasa recta capillaries in the renal medulla (see Supplementary Movie 1). Furthermore, we demonstrate that sympathetic nerve varicosities are in close apposition to pericyte cells on vasa recta (Figure 1). Interestingly, sympathetic nerve varicosities are in closer apposition to pericytes in the outer medulla rather than inner medulla (Figure 1B), perhaps supporting a less important role for sympathetic innervation of vasa recta pericytes in the inner medulla where their density is known to be 


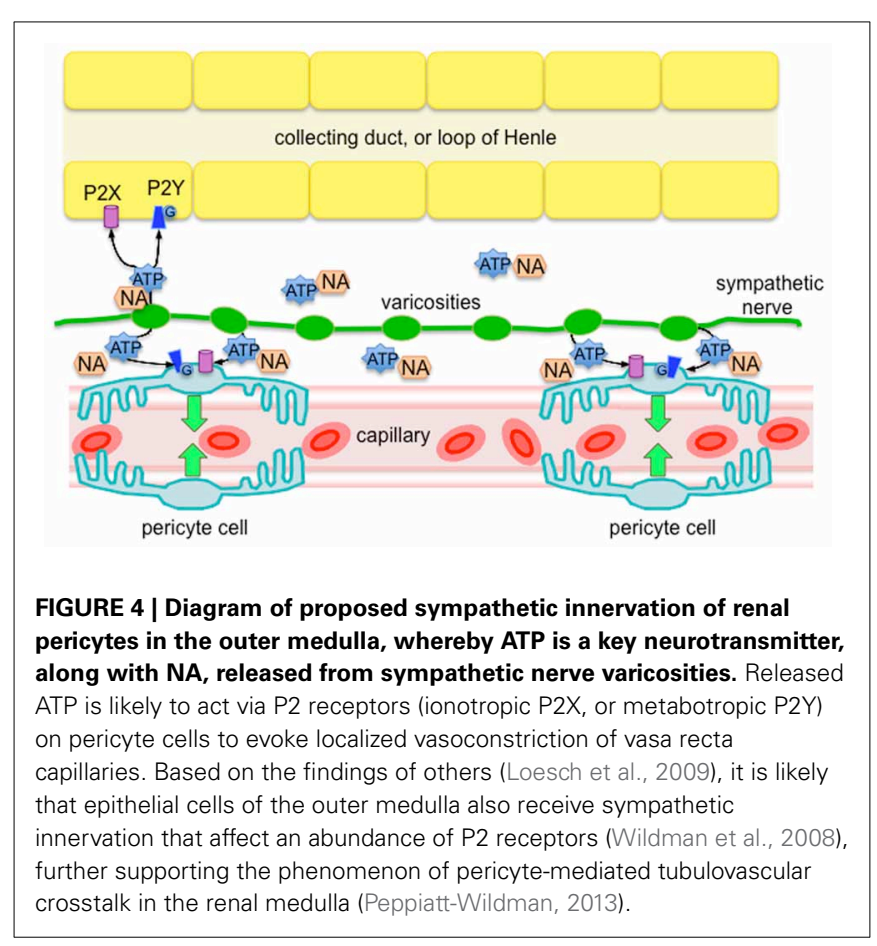

significantly less (Crawford et al., 2012). Pericyte cells were found to be $\sim 1.5 \mu \mathrm{m}$ from the nearest varicosity in the outer medulla, as opposed to $\sim 5 \mu \mathrm{m}$ in the inner medulla. Autonomic nervous system synaptic clefts, which allows delivery of co-transmitters to the site of the effector cells, vary in size with clefts size ranging from $10 \mathrm{~nm}$ up to $2 \mu \mathrm{m}$ (Burnstock, 2008). Varicosities being similar structures, whereby co-transmitters are released "en passage," likely affect effector cells in a $2 \mu \mathrm{m}$ proximity. Given the close proximity $(\sim 1.5 \mu \mathrm{m})$ of sympathetic nerve varicosities to pericytes in the outer medulla, it is therefore plausible that co-transmitters released by apposite sympathetic nerve varicosities could act at contractile vasa recta pericytes to regulate vasa recta capillary diameter and thus MBF, as hypothesized. Similarly, anti-TH antibodies have previously been used to identify sympathetic nerve varicosities in close apposition to proximal tubule and collecting duct epithelial cells of the rat kidney cortex (Loesch et al., 2009). Authors propose that sympathetic neurotransmitters (primarily ATP), released from varicosity vesicles, may regulate tubule function in the renal cortex through the activation of a variety and abundance of ATP-activated P2 receptors; shown to be expressed throughout the nephron by others (Wildman et al., 2009). In accordance with the functional data describing a key role for sympathetic nerve-derived ATP in the regulation of vasa recta diameter via contractile pericytes, we have previously reported that vasa recta and associated pericytes express mRNA for P2X1, 3, and 7 and P2Y4 and 6 (Crawford et al., 2011). Interestingly, in vivo studies in rabbits conclude that $\mathrm{P} 2 \mathrm{X}$ receptors do not contribute to neurally mediated vasoconstriction (Eppel et al., 2006a,b,c), however this is perhaps not surprising given that we have previously demonstrated much higher levels (40-fold) of $\mathrm{P} 2 \mathrm{Y}\left(\mathrm{P}_{2} \mathrm{Y}_{4}\right.$ and $\mathrm{P}_{2} \mathrm{Y}_{6}$ ) than $\mathrm{P} 2 \mathrm{X}$ receptor mRNA in rat isolated vasa recta, with pericytes in situ (Crawford et al., 2011); supporting sympathetically-derived ATP acting via P2Y receptors on pericytes to mediate vasoconstriction of vasa recta.

It is perhaps interesting to note that whilst all capillary networks are deemed to have a degree of pericyte coverage, capillaries in skeletal muscle of the rat (Saltzman et al., 1992), and peripheral blood vessels of the mouse (Long and Segal, 2009), are not innervated; suggesting that not all pericytes receive sympathetic innervation.

\section{ATP IS A KEY NEUROTRANSMITTER RELEASED FROM SYMPATHETIC VARICOSITIES}

As well as being a substrate for NA synthesis, the ability of tyramine to evoke sympathetic neurotransmitter release is well documented (Kirkpatrick and Burnstock, 1994). Notably in our preparation, the time between application of tyramine and maximum dilation of vasa recta by pericytes $(\sim 550 \mathrm{~s})$ is greater than that seen by the superfusion of a vasoactive agent like NA or ATP $(\sim 100 \mathrm{~s}$; Crawford et al., 2011, 2012). In our slice preparation it is likely that tyramine is both responsible for release, synthesis and subsequent release of newly synthesized co-transmitters (Figure 2A). Various neurotransmitters, most notably NA and ATP are contained in a single sympathetic nerve varicosity, all of which are released at one time, the proportion of each released in the current study was unknown. Pharmacological intervention, using a specific ATP-activated P2 receptor antagonist, suramin, allowed an estimation of the proportion of the tyramine-evoked response attributable to ATP released from sympathetic nerves. Tyramine-evoked vasoconstriction of vasa recta by pericytes was significantly inhibited $(\sim 50 \%)$ by the $\mathrm{P} 2$ receptor antagonist suramin (Figure 2C), suggesting a key role for sympathetic purinergic signaling in the regulation of MBF by renal pericytes. Interestingly, others have shown that the $\alpha$-adrenoceptor antagonist prazosin greatly reduces RBF and CBF in response to renal nerve stimulation, yet MBF was both reduced and increased (Chapman et al., 1982; Eppel et al., 2004, 2006a,b,c). This may of course be due to the relative expression of $\alpha$-adrenoceptors in the vasculature throughout the kidney, which was not reported by authors of these studies.

\section{SYMPATHETIC INNERVATION PROVIDES A FINE-TUNING MECHANISM}

The current study demonstrates that the vasoconstriction of vasa recta by pericytes evoked by: (i) superfusion of ATP onto live kidney slices, and (ii) tyramine-stimulated release of co-transmitters, are additive. The renal medulla contains numerous potential sources of vasoactive ATP, including from tubular cells, endothelial cells, and red blood cells (Bodin et al., 1991; Sprague et al., 1996; Schwiebert, 2001; Jans et al., 2002; Praetorius et al., 2005; Wildman et al., 2009), in addition to that released by sympathetic nerve varicosities. The additive effects of neuronal co-transmitters and paracrine-released ATP on the renal vasculature, has previously been demonstrated to enhance smooth-muscle cellmediated vasoconstriction of the glomerular afferent arteriole (Hultstrom et al., 2007). That tyramine-evoked constriction of vasa recta by pericytes is significantly less than exogenous ATPevoked effects, or indeed maximal exogenous NA-evoked effects (Crawford et al., 2011, 2012), to our minds suggest that sympathetic innervation fine-tuning of MBF can be achieved. However, 
it is noteworthy that different proportions of co-transmitter are released in different tissues and the contribution of each cotransmitter can depend on a number of parameters of stimulation, e.g., that short bursts of electrical stimulation favor ATP release, and longer bursts favor NA (Burnstock, 2007).

\section{CONCLUSIONS}

We hypothesized that sympathetic nerves are an additional source of ATP to act upon renal pericytes to therefore regulate MBF (see Figure 4). Here we present evidence to support our hypothesis; however acknowledge that in vivo studies are still required to provide direct information regarding the control of MBF. What remains to be determined is under which physiological and/or pathophysiological circumstances that sympathetic innervation of renal pericytes, and therefore MBF, is important. Like others we assume that coordination exists between sympathetic nerves and intra-renal effectors such as vessels, tubules and juxtaglomerular cells [neatly termed tubulovascular crosstalk (Dickhout et al., 2002; Kennedy-Lydon et al., 2013; Peppiatt-Wildman, 2013), see Figure 4], and that there are functionally specific subgroups of renal nerve fibers mediating specific effects on the renal tubular, vascular or juxtaglomerular granular cells, such as those involved in $\mathrm{RBF}$ response and urinary flow rate response (Dibona et al., 1996; Dibona, 2000). A better understanding of these neural sub-populations will determine the significance of renal purinergic innervation and $\mathrm{MBF}$, which is undoubtedly overlooked in light of renal transplantation (denervated kidney) successes, and misconception that kidneys have complete intrinsic ability to regulate blood flow without the need for autonomic input [despite it being well accepted that control of renal hemodynamics involves both intrinsic (myogenic and TGF components of autoregulation) and extrinsic mechanisms]. To contextualize the potential significance of these findings, considering what is now

\section{REFERENCES}

Barajas, L., Liu, L., and Powers, K. (1992). Anatomy of the renal innervation: intrarenal aspects and ganglia of origin. Can. J. Physiol. Pharmacol. 70, 735-749. doi: 10. 1139/y92-098

Bodin, P., Bailey, D., and Burnstock, G. (1991). Increased flow-induced ATP release from isolated vascular endothelial cells but not smooth muscle cells. Br. J. Pharmacol. 103, 1203-1205. doi: 10.1111/j.14765381.1991.tb12324.x

Burnstock, G. (1990). Noradrenaline and ATP as cotransmitters in sympathetic nerves. Neurochem. Int. 17, 357-368. doi: 10.1016/01970186(90)90158-P

Burnstock, G. (2007). Physiology and pathophysiology of purinergic neurotransmission. Physiol. Rev. 87, 659-797. doi: 10.1152/physrev.000 43.2006

Burnstock, G. (2008). Non-synaptic transmission at autonomic neuroeffector junctions. Neurochem. Int. 52,

known about the regulation of MBF: despite the hypoxic environment of the renal medulla, regulated $\mathrm{MBF}$ serves to provide the vascular and highly metabolic tubular cells with adequate oxygen and nutrients whilst clearing metabolic waste. Imbalances in $\mathrm{MBF}$ regulation are detrimental to the health of the kidney as a whole, with localized ischemia leading to papillary necrosis and loss of appropriate sodium and water transport in the loops of Henle. Moreover, it is well established that dysregulation of $\mathrm{MBF}$, acute or chronic, can result in significant pathology. Should sympathetically-derived ATP be involved in said dysregulation, renal pericytes, and indeed purinergic signaling pathways, may represent a novel a therapeutic target in the future.

\section{ACKNOWLEDGMENTS}

Experimental work was supported by the Medical Research Council (MRC, UK) and The Wellcome Trust (UK). We thank Miss Jessica Munday for assistance with immunohistochemistry experiments.

\section{SUPPLEMENTARY MATERIAL}

The Supplementary Material for this article can be found online at: http://www.frontiersin.org/journal/10.3389/fphys.2013.00307/ abstract

Supplementary Movie 1 | Z-stack series of images showing co-localization of pericytes and sympathetic nerves in the outer medulla. Pericytes and sympathetic nerves were labeled with anti-NG2, and tyrosine hydroxylase primary antibodies, respectively, and were subsequently probed with fluorescent Alexa-555 (red) and Alexa-488 (green)-conjugated antibodies, respectively. The z-stack series images $5.6 \mu \mathrm{m}$ through the tissue slice; sympathetic nerves can be seen to run in close proximity to pericytes on vasa recta capillaries. Scale bar $=10 \mu \mathrm{m}$.

treated with alpha-adrenergic and dopaminergic blockers. J Physiol. $325,67-77$.

Dibona, G. F. (2000). Neural control of the kidney: functionally specific renal sympathetic nerve fibers. Am. J. Physiol. Regul. Integr. Comp. Physiol. 279, R1517-R1524.

Dibona, G. F. (2005). Physiology in perspective: the wisdom of the body. Neural control of the kidney. Am. J. Physiol. Regul. Integr. Comp. Physiol. 289, R633-R641. doi: 10.1152/ajpregu.00258.2005

Dibona, G. F., Sawin, L. L., and Jones, S. Y. (1996). Differentiated sympathetic neural control of the kidney. Am. J. Physiol. 271, R84-R90.

Dickhout, J. G., Mori, T., and Cowley, A. W. Jr. (2002). Tubulovascular nitric oxide crosstalk: buffering of angiotensin II-induced medullary vasoconstriction. Circ. Res. 91, 487-493. doi: 10.1161/01.RES.000 0035243.66189 .92

Eppel, G. A., Lee, L. L., and Evans, R. G. (2004). alpha-Adrenoceptor subtypes mediating regional kidney blood flow responses to renal nerve stimulation. Auton. Neurosci. 112, 15-24. doi: 10.1016/j.autneu.2004. 03.001

Eppel, G. A., Ventura, S., Denton, K. M., and Evans, R. G. (2006a). Lack of contributionof $\mathrm{P} 2 \mathrm{X}$ receptors to neurally mediated vasoconstriction of the rabbit kidney in vivo. Acta Physiol. 186, 197-207. doi: 10.1111/j.1748-1716.2006.01526.x

Eppel, G. A., Ventura, S., and Evans, R. G. (2006b). Regional vascular responses to ATP and ATP analogues in the rabbit kidney in vivo: roles for adenosine receptors and prostanoids. Br. J. Pharmacol. 149, 523-531. doi: 10.1038/sj.bjp. 0706901

Eppel, G. A., Luff, S. E., Denton, K. M., and Evans, R. G. (2006c). Type 1 neuropeptide $\mathrm{Y}$ receptors and alphal-adrenoceptors in the neural control of regional renal perfusion. Am. J. Physiol. Regul. Integr. Comp. Physiol. 290, 
R331-R340. doi: 10.1152/ajpregu. 00317.2005

Glover, V., Pycock, C. J., and Sandler, M. (1983). Tyramine-induced noradrenaline release from rat brain slices:prevention by (-)-deprenyl. Br. J. Phramacol. 80, 141-148. doi: $10.1111 / \mathrm{j} .1476-5381.1983 . t b 1$ 1059.x

Hultstrom, M., Lai, E. Y., Ma, Z., Kallskog, O., Patzak, A., and Persson, A. E. (2007). Adenosine triphosphate increases the reactivity of the afferent arteriole to low concentrations of norepinephrine. Am. J. Physiol. Regul. Integr. Comp. Physiol. 293, R2225-R2231. doi: 10.1152/ajpregu.00287.2007

Jans, D., Srinivas, S. P., Waelkens, E., Segal, A., Lariviere, E., Simaels, J., et al. (2002). Hypotonic treatment evokes biphasic ATP release across the basolateral membrane of cultured renal epithelia (A6). J. Physiol. 545, 543-555. doi: 10.1113/jphysiol.2002.026641

Johns, E. J., Kopp, U. C., and DiBona, G. F. (2011). Neural control of renal function. Compr. Physiol. 1, 731-767. doi: 10.1002/cphy.c100043

Kennedy-Lydon, T. M., Crawford, C., Wildman, S. S., and PeppiattWildman, C. M. (2013). Renal pericytes: regulators of medullary blood flow. Acta Physiol. 207, 212-225. doi: 10.1111/apha.12026

Kirkpatrick, K. A., and Burnstock, G. (1994). Release of endogenous ATP from the vasa deferentia of the rat and guinea-pig by the indirect sympathomimetic tyramine. J. Auton. Pharmacol. 14, 325-335. doi: 10. 1111/j.1474-8673.1994.tb00613.x

Leonard, B. L., Evans, R. G., Navakatikyan, M. A., and Malpas, S. C. (2000). Differential neural control of intrarenal blood flow.
Am. J. Physiol. Regul. Integr. Comp. Physiol. 279, R907-R916.

Loesch, A., Unwin, R., Gandhi, V., and Burnstock, G. (2009). Sympathetic nerve varicosities in close apposition to basolateral membranes of collecting duct epithelial cells of rat kidney. Nephron Physiol. 113, p15-p21. doi: 10.1159/000235246

Long, J. B., and Segal, S. S. (2009). Quantifying perivascular sympathetic innervation: regional differences in male C57BL/6 mice at 3 and 20 months. J. Neurosci. Methods 184, 124-128. doi: 10. 1016/j.jneumeth.2009.07.028

Park, F., Mattson, D. L., Roberts, L. A., and Cowley, A. W. Jr. (1997). Evidence for the presence of smooth muscle alpha-actin within pericytes of the renal medulla. Am. J. Physiol. 273, R1742-R1748.

Peppiatt, C. M., Howarth, C., Mobbs, P., and Attwell, D. (2006). Bidirectional control of CNS capillary diameter by pericytes. Nature 443, 700-704. doi: 10.1038/ nature 05193

Peppiatt-Wildman, C. M. (2013). The evolving role of renal pericytes. Curr. Opin. Nephrol. Hypertens. 22, 10-16. doi: 10.1097/MNH.0b01 3e32835b4e6e

Praetorius, H. A., Frokiaer, J., and Leipziger, J. (2005). Transepithelial pressure pulses induce nucleotide release in polarized MDCK cells. Am. J. Physiol. Renal Physiol. 288, F133-F141. doi: 10.1152/ajprenal. 00238.2004

Rudenstam, J., Bergstrom, G., Taghipour, K., Gothberg, G., and Karlstrom, G. (1995). Efferent renal sympathetic nerve stimulation in vivo. Effects on regional renal haemodynamics in the Wistar rat, studied by laser-Doppler technique.
Acta Physiol. Scand. 154, 387-394. doi: $\quad 10.1111 / \mathrm{j} .1748-1716.1995 . t b$ 09922.x

Rump, L. C., Bohmann, C., Schwertfeger, E., Krumme, B., Von Kugelgen, I., and Schollmeyer, P. (1996). Extracellular ATP in the human kidney: mode of release and vascular effects. J. Auton. Pharmacol. 16, 371-375. doi: 10.1111/j.14748673.1996.tb00056.x

Saltzman, D., Delano, F. A., and Schmid-Schonbein, G. W. (1992). The microvasculature in skeletal muscle. VI. Adrenergic innervation of arterioles in normotensive and spontaneously hypertensive rats. Microvasc. Res. 44, 263-273. doi: 10.1016/0026-2862(92)90086-5

Schwiebert, E. M. (2001). ATP release mechanisms, ATP receptors and purinergic signalling along the nephron. Clin. Exp. Pharmacol. Physiol. 28, 340-350. doi: 10.1046/j. 1440-1681.2001.03451.x

Sprague, R. S., Ellsworth, M. L., Stephenson, A. H., and Lonigro, A. J. (1996). ATP: the red blood cell link to NO and local control of the pulmonary circulation. Am. J. Physiol. 271, H2717-H2722.

Trendelenberg, U., Langeloh, A., and Bonisch, H. (1987). Mechanism of action of indirectly acting sympathomimetic amines. Blood Vessels 24, 261-270. doi: 10.1159/000158702

Walkowska, A., Badzyñska, B., Kompanowska-Jezierska, E., Johns, E. J., and Sadowski, J. (2005). Effects of renal nerve stimulation on intrarenal blood flow in rats with intact or inactivated NO synthases. Acta Physiol. Scand. 183, 99-105. doi: $\quad$ 10.1111/j.1365-201X.2004. 01376.x

Wildman, S. S., Kang, E. S., and King, B. F. (2009). ENaC, renal sodium excretion and extracellular ATP. Purinergic Signall. 5, 481-489. doi: 10.1007/s11302-009-9150-6

Wildman, S. S., Marks, J., Turner, C. M., Yew-Booth, L., PeppiattWildman, C. M., King, B. F., et al. (2008). Sodium-dependent regulation of renal amiloride-sensitive currents by apical P2 receptors. J. Am. Soc. Nephrol. 19, 731-742. doi: 10.1681/ASN.2007040443

Conflict of Interest Statement: The authors declare that the research was conducted in the absence of any commercial or financial relationships that could be construed as a potential conflict of interest.

Received: 29 June 2013; accepted: 08 October 2013; published online: 29 October 2013.

Citation: Crawford C, Wildman SSP, Kelly MC, Kennedy-Lydon TM and Peppiatt-Wildman CM (2013) Sympathetic nerve-derived ATP regulates renal medullary vasa recta diameter via pericyte cells: a role for regulating medullary blood flow? Front. Physiol. 4:307. doi: 10.3389/fphys.2013.00307 This article was submitted to Renal and Epithelial Physiology, a section of the journal Frontiers in Physiology. Copyright (๑ 2013 Crawford, Wildman, Kelly, Kennedy-Lydon and PeppiattWildman. This is an open-access article distributed under the terms of the Creative Commons Attribution License (CC BY). The use, distribution or reproduction in other forums is permitted, provided the original author(s) or licensor are credited and that the original publication in this journal is cited, in accordance with accepted academic practice. No use, distribution or reproduction is permitted which does not comply with these terms. 